\title{
Colonoscopy a Real Diagnostic Paragon
}

\author{
A. Z. Kaleem, N. Naheed, S. M. Ahmad \\ Scunthorpe General Hospital, North Lincolnshire \& Goole NHS Foundation Trust, Scunthorpe, UK \\ Email: ahmed.kaleem@nhs.net
}

How to cite this paper: Kaleem, A.Z., Naheed, N. and Ahmad, S.M. (2017) Colonoscopy a Real Diagnostic Paragon. Surgical Science, 8, 256-268. https://doi.org/10.4236/ss.2017.86028

Received: February 13, 2017

Accepted: June 25, 2017

Published: June 28, 2017

Copyright (c) 2017 by authors and Scientific Research Publishing Inc. This work is licensed under the Creative Commons Attribution International License (CC BY 4.0).

http://creativecommons.org/licenses/by/4.0/

\begin{abstract}
Diagnostic colonoscopy acquires the status of gold standard investigation for evaluation of colonic problems. Formally, colonoscopy was first initiated in June 1969 in America. Colonoscopic history can be traced back to 1958. Matsunaga from Japan was the pioneer as he used a gastrocamera which was the start of colonoscopic era. Flexible sigmoidoscopy was later introduced in 1963. Existing colonic perforation and refusal of a fully compos mentis patient, remain absolute contraindications to diagnostic colonoscopy. Bowel Perforation, although less common, is a disastrous complication of colonoscopy. It is a basic right of any patient who has been offered a colonoscopic procedure to have appropriate, clear and concise information about colonoscopy. Consent literally means permission or an agreement which is granted by a patient to a health care individual to receive examination, test, treatment or intervention. Colonoscopic consent process should be valid and has voluntary, fully informed and capacity aspects as its integral components. There were a lot of oppositions against colonoscopy at that time. It was believed to be dangerous and unnecessary exercise by many surgeons. Time has proved that to be a wrong perception. It is now believed to be an extremely useful diagnostic modality. It is safe procedure in experienced hands and provides valuable clinical information. Colonoscopy is not a pleasant investigation as it is usually related with pain and discomfort by patients; fortunately, good analgesia and safe conscious sedation make this hostile but worthwhile procedure tolerable to patients. Today colonoscopy has kept its status of the gold standard investigation in diagnosing bowel cancer and has prime role in the assessment of majority of large bowel symptoms. Colonoscopy also retains the status of being an investigation with highest sensitivity and specificity, out of all available diagnostic modalities, for identifying adenomatous polyps and now it has acquired a unique status of being a diagnostic paragon.
\end{abstract}

\section{Keywords}

Diagnostic Colonoscopy, Indication, Contraindication, Complications, Colorectal cancer, Consent, Communication 


\section{Introduction}

Colonoscopic history can be traced back to 1958 in Japan where Matsunaga first used a gastro camera in the colon under fluoroscopic guidance [1]. Flexible sigmoidoscopy was later introduced in 1963 and from its start it acquired the status of gold standard investigation for evaluation of colonic problems [2]. Formally, colonoscopy was first initiated in June 1969 in America. There were a lot of oppositions against colonoscopy at that time. It was believed to be dangerous and unnecessary exercise by many surgeons. Time has proved that to be a wrong perception. It is now believed to be an extremely useful diagnostic modality. Today colonoscopy has kept its status of the gold standard investigation in diagnosing bowel cancer and has prime role in the assessment of majority of large bowel symptoms. Colonoscopy also retains the status of being an investigation with highest sensitivity and specificity, out of all available diagnostic modalities, for identifying adenomatous polyps [3].

\section{Indications}

British Society of Gastroenterology (BSG) and the Association of Coloproctology for Great Britain and Ireland (ACPGBI), in its policy statement, strongly recommends colonoscopy in the diagnosis of the following large bowel symptoms which could be suggestive of bowel cancer or adenomatous polyps [4].

\subsection{Symptoms Suggestive of Colorectal Cancer}

\section{Rectal bleeding}

Rectal bleeding could be a symptom of colorectal cancer. Any patient who is above 60 year of age and present with rectal bleeding which persist for 6 weeks or more, should be offered colonoscopy regardless of their sex. It is also recommended to offer colonoscopy to a patient who is above 40 years of age and symptomatic with rectal bleeding with associated change in his or her bowel habits persisting 6 weeks or more [4].

\section{Change in bowel habits}

Change in bowel habits can be a worrying symptom and should be investigated thoroughly with colonoscopy. Change in bowel habits persisting for 6 weeks or more without rectal bleeding, above 60 years of age patient, should have diagnostic colonoscopy according to [4].

\section{Anaemia}

Iron deficiency anaemia could be a symptom of bowel cancer and therefore unexplained iron deficiency anaemia is another indication for performing diagnostic colonoscopy.

\section{Altered blood or blood mixed in stool}

All patients who present with altered blood or blood mixed in stool should be investigated with the help of colonoscopyregardless of their age. This symptom could be also an indication of colorectal neoplasia and must not be ignored.

\subsection{Other Diagnostic Indications}

British Society of Gastroenterology (BSG) and the Association of Coloproctology 
for Great Britain and Ireland (ACPGBI) also advocate to have diagnostic colonoscopy in following conditions.

\section{Melaena}

British society of Gastroenterologist recommends colonoscopy in patients with melaena only after upper gastro intestinal cause is ruled out. It means to perform upper gastro intestinal endoscopy first and if that is found to be normal then to attempt colonoscopy and mesenteric angiogram accordingly to establish the cause of bleeding.

\section{Rectal bleeding}

Rectal bleeding in patients with emergency admission should be offered colonoscopy to establish cause.

\section{Diarrhoea}

Unexplained chronic diarrhoea is a clinical manifestation which warrants colonoscopy and sometime random biopsies as this could be related to microscopic colitis etc.

\section{Abnormal findings in colon}

All abnormal or suspicious findings in colon which are detected on barium enema, computerised tomography or virtual colonoscopy should be offered colonoscopy plus minus biopsy.

\section{Abnormalities of terminal ileum}

It is also vital to perform colonoscopy for any unexplained abnormality found in terminal ileum on magnetic resonance imaging or other small bowel imaging.

\section{Persistent abdominal symptoms}

Persistent abdominal symptoms should be properly investigated. Certain patients who present with raised $c$ reactive protein or faecalcalprotectin are best investigated with colonoscopy as inflammatory bowel disease or diverticular disease can be cause of these persistent abdominal symptoms.

\section{Inflammatory bowel disease diagnosis}

Once Inflammatory Bowel Disease (IBD) is suspected then it is crucial to perform colonoscopy for assessment of extent, activity and type of disease.

\section{Inflammatory bowel disease treatment and surveillance}

Colonoscopy has its unique role in assessing mucosal response to treatment with medical therapy in patients with Crohn's disease. Colonoscopy is also recommended for surveillance purposes in patients who suffer with IBD.

\section{Screening}

National bowel cancer screening programme is mainly based on colonoscopy investigation. Certain age group individuals are offered faecal occult blood testing first in this programme. Then subjects with positive faecal occult blood tests are referred for colonoscopy by a bowel screener colonoscopists, to rule out any polyps or cancers. Individual with significant family history of bowel cancer are also offered screening with colonoscopy.

\section{Surveillance}

Colonoscopic surveillance is advocated in patients with familial adenomatous polyposis (FAP). There are specific guidelines available about timing of perform- 
ing colonoscopy after resection of colorectal cancer and removal of adenomas.

\section{Abnormal findings in flexible sigmoidoscopy}

Synchronous neoplasms or polyps are always cause of concern therefore, after finding any abnormality (Polyp/Cancer) in flexible sigmoidoscopy, it is wise to perform complete colonoscopy to rule out any synchronous lesion.

\section{Contraindications}

Majority of the times, colonoscopic procedures can be performed safely but there are occasions, when colonoscopy is deemed as dangerous exercise and can lead to further harm to the patient. These occasions are broadly classified in to absolute contraindications and relative contraindications.

\section{Absolute contraindications}

Absolute contraindications to colonoscopy include the following two conditions [2].

\section{Refusal of a patient}

This category comprises of a patient who has demonstrated full capacity and refuses to undergo colonoscopic procedure after being fully informed.

\section{Colonic perforation}

Patient with existing colonic perforation should not undergo colonoscopy as this could lead to further harm to the patient.

\section{Relative contraindications}

There are also some conditions which are termed as relative contraindications where colonoscopy is considered as relatively unsafe procedure.

\section{Acute diverticulitis}

Colonoscopy in acute diverticulitis should be avoided as this could lead to colonic perforation. This complication could be life threatening and might need urgent operative management, therefore it is unsafe exercise and not recommended.

\section{Immediately post-operative patients}

Colonoscopy is relatively contraindicated in immediately post-operative patients for obvious reasons. This relative contraindication does depend on the nature of surgery as minor surgical procedures are not included in this category.

\section{Recent myocardial infarction}

Colonoscopy is relatively contraindicated for first three months after myocardial infarction [1]. There is also suggestion to avoid colonoscopy at least in first month after myocardial infarction in the literature [2], but is best to be avoided for at least three months as colonoscopy at that stage could lead to lethal cardiac dysthymias.

\section{Pulmonary embolism}

This is also considered as a relative contraindication in the immediate post event phase. Patient with pulmonary embolism may require prior haematologist advice in the late post event period.

\section{Hemodynamic instability}

This extremely unwell patient's category needs to be stabilised first before 
proceeding to any invasive colonoscopic procedure. Sometime endoscopy can be performed along with resuscitation, in unstable patients who are suffering from disastrous upper GI haemorrhage, as this procedure is deemed to be lifesaving in that challenging situation.

\section{Severe coagulopathy}

Severe coagulopathy needs to be corrected prior to colonoscopy, as this could lead to catastrophic bleeding.

\section{Ascites/Peritoneal dialysis}

In this group of patients, colonoscope due to its pressure effect, can lead to transient release of bowel organism in to peritoneal cavity or blood stream and this eventually lead to bacteraemia or septicaemia [1]. Therefore, colonoscopy is not recommended.

It is also noted that colonoscopy can safely be performed in pregnancy but preferably delayed unless there is a strong indication for immediate examination [2].

\section{Complications}

The term complication is strongly associated with complaint process and has acquired medicolegal importance. Therefore, it is important to fully inform the patient, who is undergoing through colonoscopic procedure, about all the possible complications of colonoscopy. Also, it is paramount to act fast once a complication is recognised and patient should be fully informed as a duty of candour set by General Medical Council.

A complication should have following three attributes [1], shown in Figure 1.

Colonoscopy is an invasive diagnostic modality which is related with many complications. Fortunately, the incidence of these complication is fairly low thus making this procedure as relatively safe procedure.

The following are the main specific complications associate with diagnostic colonoscopy.

\subsection{Perforation}

This is a disastrous complication of colonoscopy. Diagnostic colonoscopy is associated to have low perforation rate between $0.016 \%$ to $0.2 \%$ as compared to

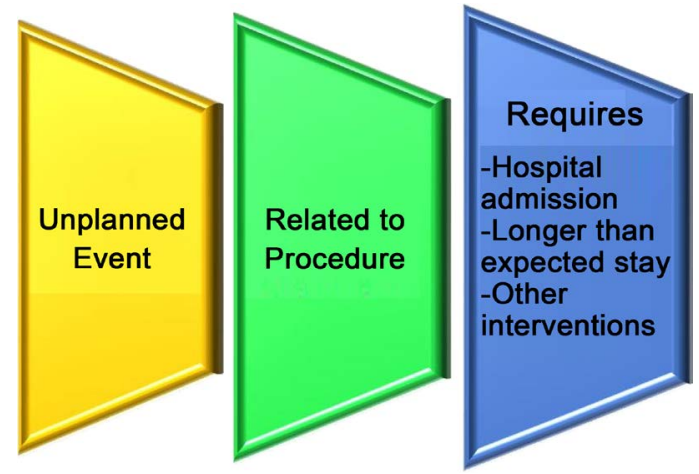

Figure 1. Complication Attributes [1]. 
therapeutic colonoscopy [5].

Historically it was thought that the incidence rate of perforation due to diagnostic colonoscopy is 1 in 1500 colonoscopic procedure as compared to 1.25000 perforation which is associated with barium studies and Ct Pneumocolon [1]. The comparative barium studies and Ct Pneumocolon is still inferior to traditional colonoscopy as these modalities lack the specialised therapeutic aspect which is only attached to colonoscopy.

Perforation in diagnostic colonoscopy could be of 2 main types, instrument tip/shaft perforation or air pressure perforation [1].

\section{Instrument tip or shaft perforation}

This usually occurs with excessive force in unexperienced hands. It was found that shaft related perforations are larger than expected as compared to tip perforations, therefore requiring operative management in majority of involved cases.

\section{Air pressure perforation}

This is a usual consequence of blow out of weak diverticulum occurs secondary to air pressure. Occasionally, these types of perforations can be managed conservatively. Sometime operative management is essential therefore, regular assessment of these patients is crucial.

\subsection{Haemorrhage}

This is also a known complication of colonoscopy. Therapeutic procedures tend to have more haemorrhage related complications as compared to diagnostic procedures. In USA, haemorrhage incidence was reported as 3.7 per 1000 for all diagnostic colonoscopic procedures [6]. The documented haemorrhage rate in diagnostic colonoscopy is found to be 1 in 500 [3] in UK.

It is paramount to make all the possible efforts to reduce this risk. Patients who are on anticoagulation should be identified early with a robust system and they should be advised accordingly. There should be local guidelines available to formulate appropriate plan to help this group of subjects. In case of bleeding, patients should be admitted for regular observation and appropriate advice should be taken from haematologist.

\subsection{Hypotensive Episodes}

Vigorous and prolong colonoscopy can lead to intense vagal stimulation. This situation can be combined with over sedation of the patient and thus can provoke a lethal hypotensive episode. This could potentially lead to respiratory or cardiac arrest. Hence, it is vital to first avoid this life-threatening situation and if at all this does happen, then to be fully prepared to deal this catastrophic condition.

\section{Communication}

It is a basic right of any patient who has been offered a colonoscopic procedure to have appropriate, clear and concise information about colonoscopy. This can only be accomplished by a health care individual with his effective communica- 
tion. Conveying and gathering of information are two fundamental components of communication. Although there is a diverse spectrum of communication but mainly there are 2 ways of communication.

\subsection{Active}

This includes one to one meeting. This information provision process starts in outpatient consultation whereby a health care provider offers colonoscopy to a patient. Later on further information is provided by nurse-specialist or pre assessment endoscopy nurse, which is a novel way and must be utilized to improve effective communication and to answer any unanswered questions.

\subsection{Passive}

This communication process mainly comprises of written information sources like leaflets, posters and available information sheets. It is advisable to provide both verbal and written information at the time of consultation. All written information should address at least these following four important key aspects (Figure 2).

The author believes that a good communicator is always a knowledgeable person who has decent command on his subject. Therefore, it is important for everyone who is providing key information to patients and carers to be well informed about colonoscopy. This learning exercise will eventually aim to provide clear, concise information to patients and carers so that they can make an informed decision in their best interest. Information should always be delivered to patients using well organized and unambiguous methods of communication.

The author has strong faith in in the work of a leading psychologist Mehrabian [8] who presented an effective theory of communication. He determined that communication can be made up of three key aspects (Figure 3).

\section{Body language}

Body language carries 55\% weightage in any communication which is one of the largest proportion. Thus body language has acquired a prime importance and should not be neglected at any time when providing colonoscopic related information.

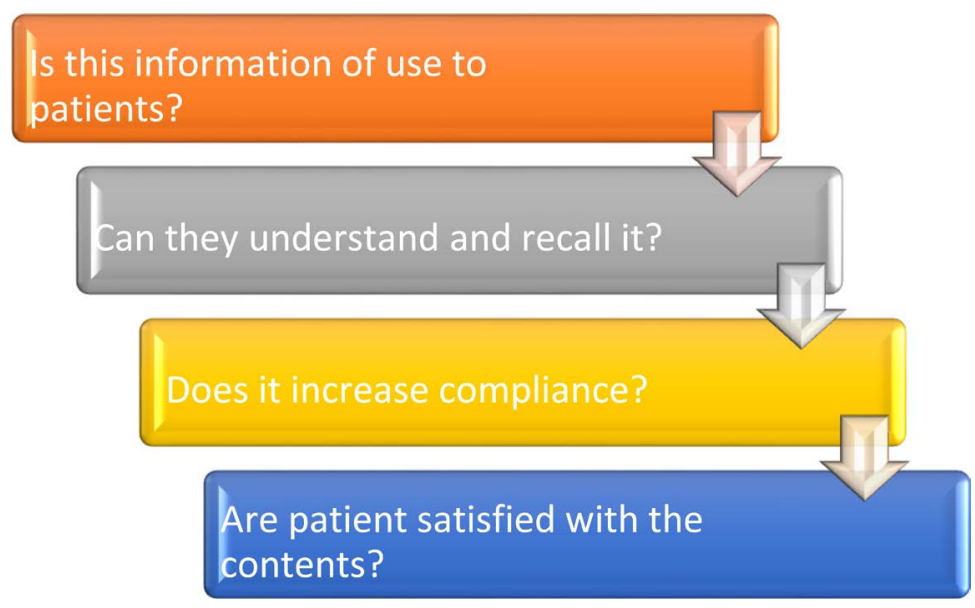

Figure 2. Patient information properties [7]. 


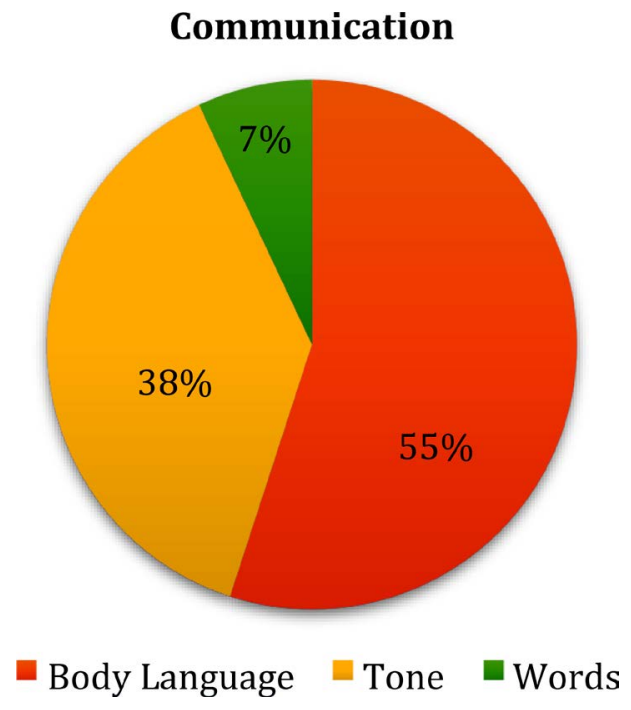

Figure 3. Mehrabian Communication Chart [8].

\section{Tone}

Tone is also vital as it carries $38 \%$ of the total share in any communication. Right tone will lead to an effective communication and bad tone can result in to a communication disaster.

\section{Words}

Words carry only 7\% power therefore, only written colonoscopic related information is not enough, as it lacks emotions and tone.

Carefully chosen words with appropriate emotions and right tone is the foundation to relay message across effectively.

It is paramount to observe the following communication principles during the process of colonoscopic associated information dissemination to a patient as recommended by British society of Gastroenterology in its recent guidelines [9].

\section{Information format}

Any colonoscopic related information should be provided in an easy, clear and understandable format. All expected pros and cons should be well informed to patients, along with available alternatives of any suggested procedure.

\section{Verbal and written information}

Both verbal and written information should be provided in all outpatient elective procedures, by the health care individual recommending the colonoscopy at the time of the consultation meeting and this should be documented in the patient's hospital notes.

\section{Sufficient time}

Sufficient time should be provided to all those patients, who are going through this invasive procedure, to make informed decision therefore, it is vital to provide all relevant information pertaining to colonoscopy well advance before the actual procedure day.

\section{Information leaflets}

All endoscopy units should have regularly updated version of colonoscopy leaflets readily available and these leaflets must be revised annually. These leaf- 
lets should also include frequently asked questions section for patients.

\section{Information leaflets language}

Common language of the local Community should be used in these information leaflets for obvious reasons.

\section{High risk patients}

High risk patients with significant co morbidities or frailty should also have detailed discussion along with written information, before the procedure.

\section{Individual concerns}

All colonoscopy associated health care individuals should promote a healthy culture which encourages any individual, who is going through colonoscopy, to express their concerns. This should also be reflected in their consent with proper documentation.

\section{Inpatient information}

In patients should also be provided with written information in easy and clear format. This should ideally be achieved well before they leave the ward for the procedure, which allow them to have sufficient thinking time to make up their mind. All efforts should be made to answer their questions.

\section{Patient declining information}

There will be few patients who do not wish to have detailed information about colonoscopy. This should be respected but it is essential to provide at least the minimum information which should comprise of the objectives of the proposed investigation or treatment, the expected level of pain or uneasiness and steps taken to lessen it. There should be proper documentation of this consultation.

\section{Information pathways}

It is stressed that all those NHS trusts which accept patients direct for colonoscopy from primary care should have local pathways and guidance available to ensure that the referral is suitable for this service and the patient is well informed.

\section{Consent Process of Colonoscopy}

It is a basic right of a patient to be provided with all the necessary information which can help in their decision-making process. Consent in medical terminology means a permission or an agreement which is granted by a patient to a health care individual to receive examination, test, treatment or intervention. Consent should be made based on provided information by a health care individual. General Medical council guideline states.

A doctor must be satisfied that they have consent (or other valid authority) before:

- Carrying out any examination or investigation

- Providing treatment or

- Involving patients in teaching or research.

Consent can be either in written format or in verbal way.

\subsection{Verbal Consent}

Verbal consent is only sufficient in certain cases. This does include carrying out an 
examination or taking bloods or performing an $\mathrm{x}$-ray etc. Sometime certain gestures, like holding out arm for blood test, are considered as indication of consent but it is imperative to ensure that patient has appropriate rationale and understanding about a procedure before considering his verbal consent as lawful.

\subsection{Written Consent}

All invasive procedures should have written consent. This does include colonoscopy because of its invasive nature and associated potential risks as recommended by British society Of Gastroenterology in its 2016 guidelines [9]. Only exception is an emergency where written consent is not always possible and compulsory.

The person who is responsible for care should take that consent. British society Of Gastroenterology recommends that consent should be obtained by the endoscopist or delegated to a suitably trained individual [9]. Although this process of consent can be delegated, but it remains the ultimate obligation of the colonoscopists to safeguard the appropriateness of consent acquisition as he is the one who is performing the procedure.

It is also mandatory to provide enough time and information to patients so that they can make decision in their best interest without any external influence.

As per National health services guideline it is imperative for any consent to have 3 key components to achieve a status of being a valid consent (Figure 4).

\subsection{Voluntary}

The ideology behind this key component is that the verdict to either consent or not to consent should only be a voluntary decision of a person which should be completely uninfluenced from any pressure from family, friends or medical staff.

\subsection{Informed}

This aspect covers all the necessary information provision to a patient. This information should include both advantages and disadvantages of receiving a treatment. A person should be informed about alternative treatments methods. Lastly it is vital to provide facts and consequences of not receiving any treatment at all.

\subsection{Capacity}

A person should be fully compos mentis. The person must demonstrate capacity

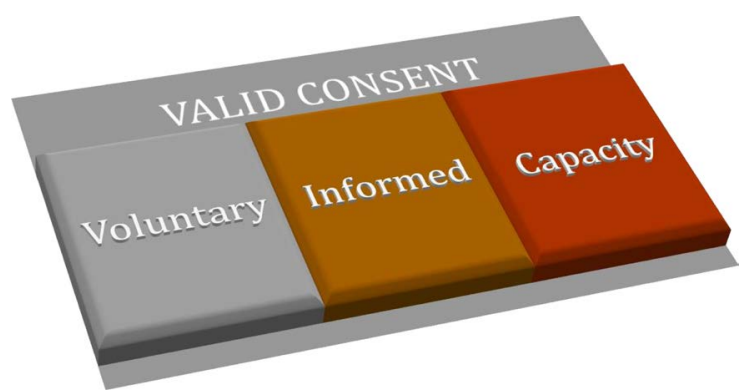

Figure 4. Valid consent (NHS). 
of giving consent which means he understands the information, retains the information fully and can use this information, given to them, to make an informed decision in their best interest. British society Of Gastroenterology in its 2016 guidelines [9] recommends that it is the duty of endoscopist performing the procedure to assess and to confirm consent capacity of his patient. Sometime the reassessment is advocated, if capacity seems to have altered since consent was first attained. It is not unwise to delay the procedure to allow further changes or to wait for the prospect to recuperate capacity.

According to British Society of Gastroenterology, It is vital for all endoscopist to demonstrate sufficient Knowledge of the law on capacity which included orientation with independent mental capacity advocates (IMCAs), familiarity with lasting powers of attorney (LPA), and advance decisions to refuse treatment law [9]. If patient lacks capacity then next point of action, whether to perform colonoscopy or not, should be always in patient's best interest.

\section{Consent in young individuals and children}

The following guidelines should always be in practice as recommended by British Society of Gastroenterology in its 2016 guidelines [9].

\section{Young people aged 16 - 18 years}

This group of young individuals are acknowledged to have the capacity to consent to endoscopy and related procedures. Endoscopists should apply similar principles like they do in adults to assess formal capacity in this group.

Competent children aged $<16$ years

Children belonging to this group can also consent to colonoscopy. Again, competence level should be assessed and confirmed by colonoscopists.

\section{Incompetent children aged $<16$ years}

This group of children cannot consent to colonoscopy as they lack capacity therefore, parental consent is required in this group.

The author strongly suggests to acquire expert legal advice where any uncertainty or issue exists about consenting to colonoscopy in a young person $(<18$ years). The same standard should be applied if the young patient declines colonoscopy.

The author practices all above mentioned principles in obtaining colonoscopy consent. It is recommended to provide details about all the possible complications of colonoscopy. Certainly, any possible complication with an incidence of 1.100 or 1.200 should never be missed in the consent part [1].

\subsection{Consent Model}

The author feels that colonoscopy consent model which has been provided by [7] is extremely useful and practical way of achieving a valid consent (Figure 5). The consent process in this model has been divided in four stages.

\section{Stage 1 Consultation}

The consent process starts in this stage. This is that initial consultation of a patient with a medical expert where he is offered colonoscopy. Ideally all the relevant information pertaining to colonoscopy is verbally given to the patient. 


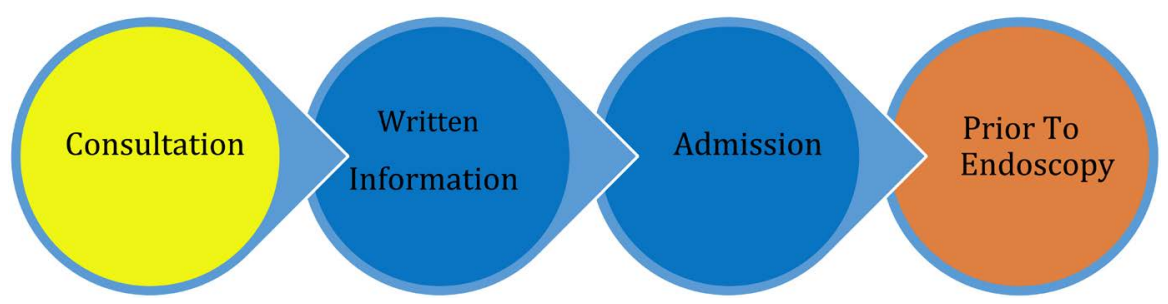

Figure 5. Colonoscopy Consent Model [7].

\section{Stage 2 Written information}

patient is provided with all the written information related to colonoscopy including information leaflets and pre-procedure preparation guidance in this stage. This stage normally take place few days before the procedure so that enough time is provided to help a patient in making his decision based on this information. Patient is also informed that he or she will not lose control at any stage of colonoscopy and has the power to withdraw consent at any time.

\section{Stage 3 Admission}

In stage 3 of consent, patient gets admitted in a ward or endoscopy unit where a qualified endoscopy nurse reiterates all the necessary information and can acquire written consent if patient is happy at that stage.

\section{Stage 4 Prior to endoscopy}

This is the final stage of consent process where endoscopist checks patient's understanding. He also offers to answer any queries and obtain written consent only if patient is fully satisfied at that stage.

As per British Society of Gastroenterology, the official consent process should be accomplished before entry into the procedure room. Final authentication of that process should happen before the procedure starts [9].

\section{Summary}

The author strongly recommends following all the above discussed guidelines to achieve risk free colonoscopic procedure which leads to patient safety and better outcome.

Colonoscopy is an extremely useful investigation which is routinely carried out to evaluate different colorectal diseases. It is safe procedure in experienced hands and provides valuable clinical information. Colonoscopy is not a pleasant investigation as it is usually related with pain and discomfort by patients; fortunately, good analgesia and safe conscious sedation make this hostile but worthwhile procedure tolerable to patients.

\section{References}

[1] Haycock, A., Cohen, J., Saunders, B.P., Cotton, P.B. and Williams, C. B. (2013) Cotton and Williams' Practical Gastrointestinal Endoscopy, John Wiley \& Sons, Oxford.

[2] Phillips, R.K. and Clark, S. (2013) Colorectal Surgery: Companion to Specialist Surgical Practice, Elsevier Health Sciences, Amsterdam.

[3] Floch, M.H. (2014) Yamada's Handbook of Gastroenterology. Journal of Clinical 
Gastroenterology, 48, 97. https://doi.org/10.1097/MCG.0b013e3182a4dfde

[4] BSG, A.A.A. (2013) Guidance on the Indications for Diagnostic Upper GI Endoscopy, Flexible Sigmoidoscopy and Colonoscopy. British Society of Gastroenterology, London.

[5] Lohsiriwat, V. (2010) Colonoscopic Perforation: Incidence, Risk Factors, Management and Outcome. World Journal of Gastroenterology (WJG), 16, 425. https://doi.org/10.3748/wjg.v16.i4.425

[6] Fisher, D.A., Maple, J.T., Ben-Menachem, T., Cash, B.D., Decker, G.A., Early, D.S., Evans, J.A., Fanelli, R.D., Fukami, N. and Hwang, J.H. (2011) Complications of Colonoscopy. Gastrointestinal Endoscopy, 74, 745-752. https://doi.org/10.1016/j.gie.2011.07.025

[7] Dalton, H., Hussaini, S., Murray, I. and Wong, J. (2002) Clinic Handbook: Gastroenterology. Taylor and Francis, Didcot.

[8] Mehrabian, A. (1970) Tactics of Social Influence. Prentice-Hall, Inc., Englewood Cliffs, New Jersey.

[9] Everett, S.M., Griffiths, H., Nandasoma, U., Ayres, K., Bell, G., Cohen, M., Thomas-Gibson, S., Thomson, M. and Naylor, K.M. (2016) Guideline for Obtaining Valid Consent for Gastrointestinal Endoscopy Procedures. Gut, 65. http://dx.doi.org/10.1136/gutjnl-2016-311904.

\section{Submit or recommend next manuscript to SCIRP and we will provide best} service for you:

Accepting pre-submission inquiries through Email, Facebook, LinkedIn, Twitter, etc. A wide selection of journals (inclusive of 9 subjects, more than 200 journals) Providing 24-hour high-quality service User-friendly online submission system Fair and swift peer-review system Efficient typesetting and proofreading procedure Display of the result of downloads and visits, as well as the number of cited articles Maximum dissemination of your research work

Submit your manuscript at: http://papersubmission.scirp.org/ Or contact ss@scirp.org 